\title{
Synthesis and Magnetic Properties of Hematite Particles in a "Nanomedusa" Morphology
}

\author{
Jin Bae Lee, ${ }^{1}$ Hae Jin Kim, ${ }^{1}$ Janez Lužnik, ${ }^{2}$ Andreja Jelen, ${ }^{2}$ Damir Pajić, ${ }^{3}$ \\ Magdalena Wencka, ${ }^{4}$ Zvonko Jagličić, ${ }^{5}$ Anton Meden, ${ }^{6}$ and Janez Dolinšek ${ }^{2}$ \\ ${ }^{1}$ Division of Materials Science, Korea Basic Science Institute, Daejeon 305-333, Republic of Korea \\ ${ }^{2}$ Faculty of Mathematics and Physics, J. Stefan Institute and University of Ljubljana, Jamova 39, SI-1000 Ljubljana, Slovenia \\ ${ }^{3}$ Department of Physics, Faculty of Science, University of Zagreb, Bijenička 32, 10000 Zagreb, Croatia \\ ${ }^{4}$ Institute of Molecular Physics, Polish Academy of Sciences, Smoluchowskiego 17, 60-179 Poznań, Poland \\ ${ }^{5}$ Faculty of Civil and Geodetic Engineering, Institute of Mathematics, Physics and Mechanics and University of Ljubljana, \\ Jamova 2, SI-1000 Ljubljana, Slovenia \\ ${ }^{6}$ Faculty of Chemistry and Chemical Technology, University of Ljubljana, Aškerčeva 5, SI-1000 Ljubljana, Slovenia
}

Correspondence should be addressed to Hae Jin Kim; hansol@kbsi.re.kr and Janez Dolinšek; jani.dolinsek@ijs.si

Received 22 August 2014; Revised 10 November 2014; Accepted 10 November 2014; Published 20 November 2014

Academic Editor: Vladimir Sivakov

Copyright (C) 2014 Jin Bae Lee et al. This is an open access article distributed under the Creative Commons Attribution License, which permits unrestricted use, distribution, and reproduction in any medium, provided the original work is properly cited.

\begin{abstract}
We present the synthesis, characterization, and magnetic properties of hematite particles in a peculiar "nanomedusa" morphology. The particles were prepared from an iron-silica complex by a hydrothermal process in a solution consisting of ethyl acetate and ethanol. The particles' morphology, structure, and chemical composition were investigated by transmission electron microscopy, powder X-ray diffraction, and scanning electron microscope equipped with an energy-dispersive X-ray spectrometer. The "hairy" particles consist of a spherical-like core of about $100 \mathrm{~nm}$ diameter and fibrous exterior composed of thin "legs" of $5 \mathrm{~nm}$ diameter grown along one preferential direction. The particles' cores are crystalline and undergo a magnetic phase transition to a weakly ferromagnetic state at a temperature of $930 \mathrm{~K}$ that matches reasonably the Néel temperature of bulk hematite. However, unlike bulk hematite that undergoes Morin transition to an antiferromagnetic state around room temperature and small hematite nanoparticles that are superparamagnetic, the "nanomedusa" particles remain weakly ferromagnetic down to the lowest investigated temperature of $2 \mathrm{~K}$. Each particle thus represents a nanodimensional "hairy" ferromagnet in a very broad temperature interval, extending much above the room temperature. Such high-temperature ferromagnetic nanoparticles are not frequently found among the nanomaterials.
\end{abstract}

\section{Introduction}

Hematite [1] $\left(\alpha-\mathrm{Fe}_{2} \mathrm{O}_{3}\right)$ is the most stable iron oxide in air under ambient conditions. It is widely used as a coloring agent (pigment) for paints and the construction industry, as a catalyst for industrial syntheses, and as one of the raw materials for the iron and steel industry. Hematite is also used in sensors for the detection of hydrocarbon gasses and carbon monoxide. Due to magnetic character and biocompatibility, hematite nanoparticles find medical applications in oncology and as magnetic contrast agents.

Hematite is commonly found in nature and can also be produced synthetically in the macrocrystalline and nanocrystalline state. Two common ways of producing crystalline hematite particles in aqueous systems are via ferrihydrite in weakly acid to alkaline media and by the hydrolysis of $\mathrm{Fe}^{\mathrm{III}}$ salt solutions at low $\mathrm{pH}$ and at elevated temperature, the socalled "forced hydrolysis method." These methods, as well as other hematite synthesis methods, are reviewed elsewhere (see, Ch. 20.2.7 of [1] and references therein). Hematite particles can be produced in a variety of morphologies, including plates and discs, rods, spindles, spheres, ellipsoids, double ellipsoids, rhombohedra, stars, cubes, and peanuts (see, for a review, Ch. 4.2.5 of [1] and references therein). Each morphology can be obtained by more than one synthetic route. In the forced hydrolysis, unusual morphologies may be produced using mixed solvents: water/ethanol or water/ethylene 
glycol $[2,3]$. Here we present the synthesis, characterization, and magnetic properties of hematite nanoparticles of peculiar morphology, consisting of a spherical-like central "body" of about $100 \mathrm{~nm}$ diameter covered by thin "legs" (fibers) of about $5 \mathrm{~nm}$ diameter, grown along one preferential direction. This shape makes the nanoparticles to resemble morphologically certain organisms from the biological world on the nanometric scale like nanobugs and nano-Cephalopoda (e.g., nanomedusa). In the following we shall refer to these particles as the hematite "nanomedusa" (HNM) particles. We demonstrate that the HNM particles undergo a magnetic phase transition to a weakly ferromagnetic state at $930 \mathrm{~K}$ and remain ferromagnetic down to the lowest investigated temperature of $2 \mathrm{~K}$, representing nanodimensional ferromagnets in a very broad temperature interval.

\section{Materials and Methods}

2.1. Material Synthesis. The HNM particles were prepared from an iron-silica complex by a hydrothermal process in a solution consisting of ethyl acetate and ethanol. $\mathrm{Fe}\left(\mathrm{NO}_{3}\right)_{3} \cdot 9 \mathrm{H}_{2} \mathrm{O}(0.81 \mathrm{~g})$ was dissolved in $25 \mathrm{~mL}$ of ethyl acetate and ethanol solvent system. $\mathrm{Na}_{2} \mathrm{SiO}_{3}(5 \mathrm{~mL}, 0.5 \mathrm{M})$ was added for the formation of silicate complex. After stirring for $30 \mathrm{mins}$, the ammonia solution $(0.05 \mathrm{~g}, 25 \%)$ was added into the upper solution to adjust to $\mathrm{pH}$ 7. The resulting suspension was then transferred into a $50 \mathrm{~mL}$ Teflon-lined autoclave with a stainless steel shell. The autoclave was kept at $185^{\circ} \mathrm{C}$ for $48 \mathrm{hrs}$ and cooled down to room temperature naturally. The so-obtained precipitates resulted in a red powder, which was washed repeatedly with distilled water and finally dried at $70^{\circ} \mathrm{C}$ in air.

2.2. Experimental Methods. The morphology of the product was examined using field-emission transmission electron microscope (FE-TEM JEOL JEM2100F). The X-ray powder diffraction (XRD) pattern was collected on an X'Pert PRO MPD diffractometer equipped with primary monochromator (pure $\mathrm{Cu} \mathrm{K} \alpha_{1}$ radiation, $\lambda=1.54056 \AA$ ). Scanning electron microscopy (SEM) was performed on a Supra VP 35 Zeiss microscope equipped with an energy-dispersive X-ray spectrometer (EDS). Magnetic properties were investigated on a Quantum Design MPMS XL-5 SQUID magnetometer equipped with a $50 \mathrm{kOe}$ magnet, operating in the temperature range between 400 and $2 \mathrm{~K}$, whereas the high-temperature data between 400 and $1000 \mathrm{~K}$ were collected on a VSM magnetometer equipped with a $10 \mathrm{kOe}$ electromagnet.

\section{Results and Discussion}

3.1. Electron Microscopy and XRD Characterization. TEM images (Figures 1(a), 1(b), and 1(c)) reveal "hairy" particles consisting of a spherical-like core of about $100 \mathrm{~nm}$ diameter and fibrous exterior composed of thin fibers of $5 \mathrm{~nm}$ diameter grown along one preferential direction. The nanoparticles are quite uniform in size. The fibers are shown on an expanded scale in Figure 1(d). A high-resolution transmission electron microscopy (HR-TEM) image, taken with atomic resolution at the outer part of the nanoparticle (Figure 1(e)), reveals the existence of oval-shaped crystalline regions of different crystallographic orientations and different cross dimensions ranging from small (about $5 \mathrm{~nm}$ ) to large (several $10 \mathrm{~nm}$ ). These crystalline regions are separated by amorphous interfaces. The small nanoparticles (light-grey color) observed, for example, in Figure 1(c), as a background to the HNM particles are amorphous $\mathrm{SiO}_{2}$, as will be demonstrated later. The electron diffraction pattern is shown in Figure 1(f), where an amorphous ring (light-grey ring in the middle of the pattern, marked by a yellow cross) due to shortrange order of the local environment is observed in addition to the sharp crystalline reflections. The amorphous pattern originates from both the amorphous $\mathrm{SiO}_{2}$ nanoparticles and the amorphous interfaces between the crystalline regions in the HNM particles.

The experimental XRD pattern of the synthesized product consisting of the HNM particles and the small "background" particles (orange trace in Figure 2(a)) shows sharp diffraction peaks characteristic of a crystalline material. All diffraction peaks could be indexed to hematite with a hexagonal corundum-type crystal structure of space group $R \overline{3} c$ and are attributed to the HNM particles. The Miller indices of the diffraction peaks are given in the diffractogram. The calculated lattice constants $a=5.032(1) \AA$ and $c=13.81(1) \AA$ match rather well the reported values for bulk hematite [4] $(a=5.038(2) \AA$ and $c=13.776(7) \AA)$, so that the unit cell of the crystalline regions in the HNM particles is to a good approximation not expanded with respect to bulk crystals. The shape of the diffraction peaks that are composed of a narrow line and a broad foot indicates a distinctive bimodal distribution of large and small crystalline domains. This distribution was determined by Rietveld analysis of the experimental XRD pattern using the structural model of hematite by Sadykov et al. [4]. The shape of the diffraction peaks was described within the "fundamental parameters approach" [5], where the adjustable parameter was the size of the crystalline domains. We assumed coexistence of two phases in the HNM particles, both hematites with the same size of the unit cell, but different size of the crystalline domains. The theoretical XRD patterns of the two phases are shown separately in Figure 2(b), where the narrow peaks (red curve) are associated with large crystalline domains, whereas the broad peaks (green curve) describe small domains. The calculated peak positions from the fitted model are shown as a stick pattern at the bottom of Figure 2(b). The sum of the two theoretical patterns is shown in Figure 2(a) by the black curve, whereas the difference between the experimental and the total theoretical pattern is shown by the cyan trace. Though the weighted $R$-factor was still relatively large, $R_{w p}=14 \%$ (mainly due to the relatively large noise, since the detector sensitivity had to be reduced because of fluorescence), the flat difference curve confirms that the model with a bimodal distribution of the crystalline domain sizes within the HNM particles fits well the experimental XRD pattern. The fit procedure yielded the large-domain average size of $89 \pm 4 \mathrm{~nm}$ and the small-domain average size 


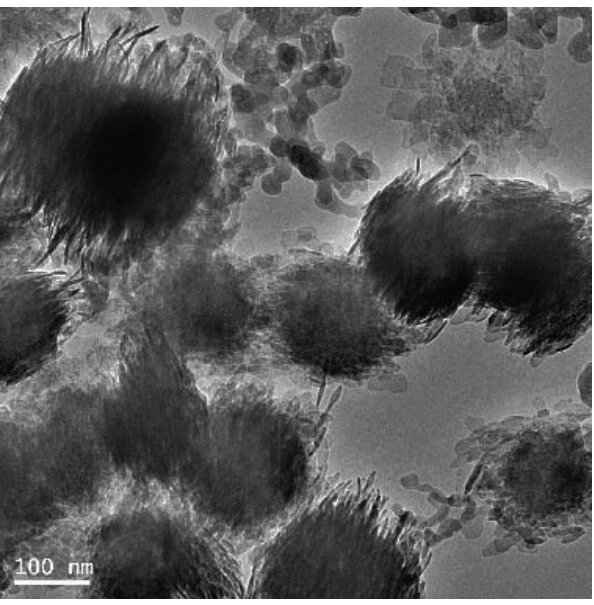

(a)

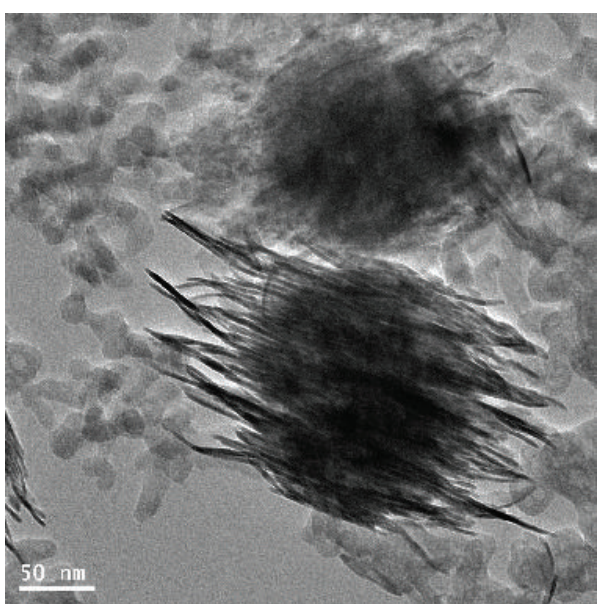

(c)

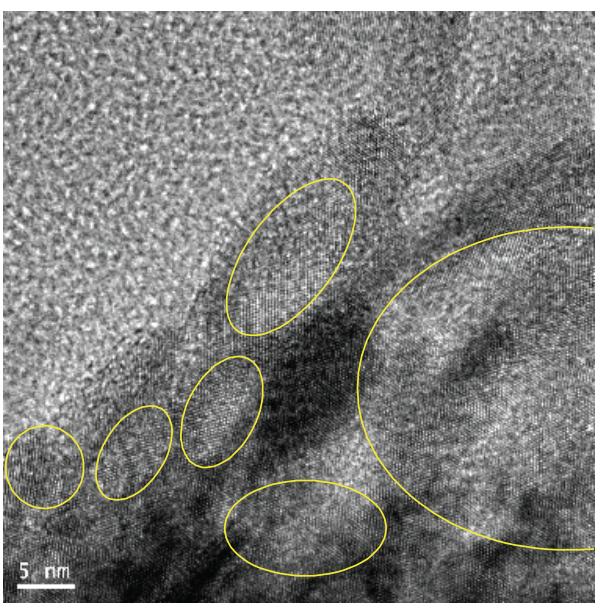

(e)

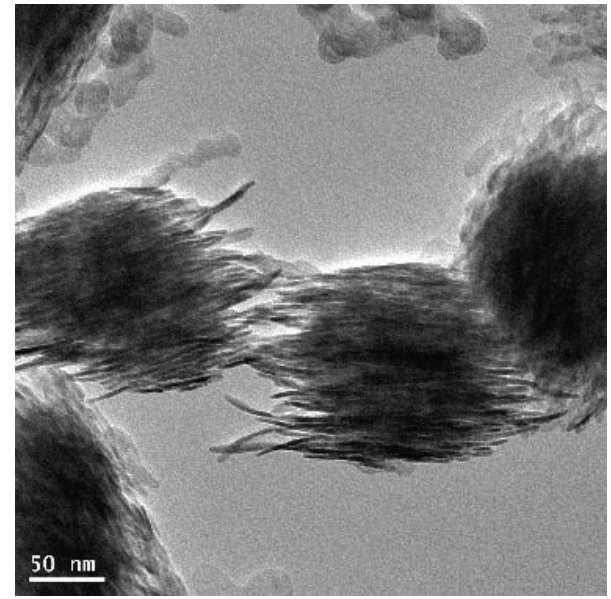

(b)

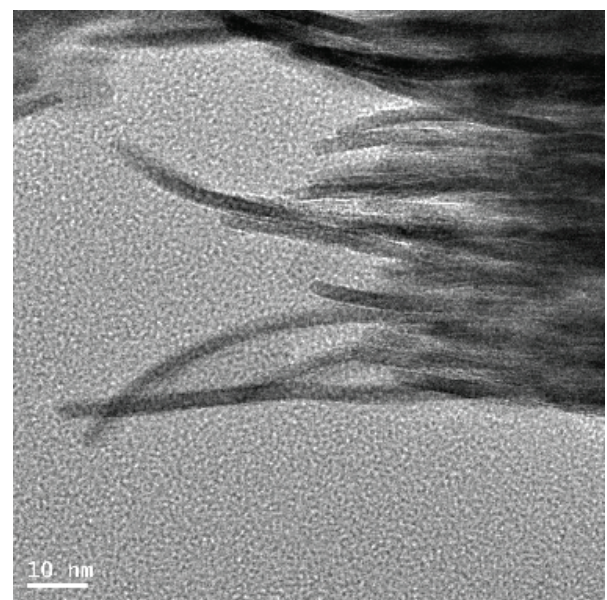

(d)

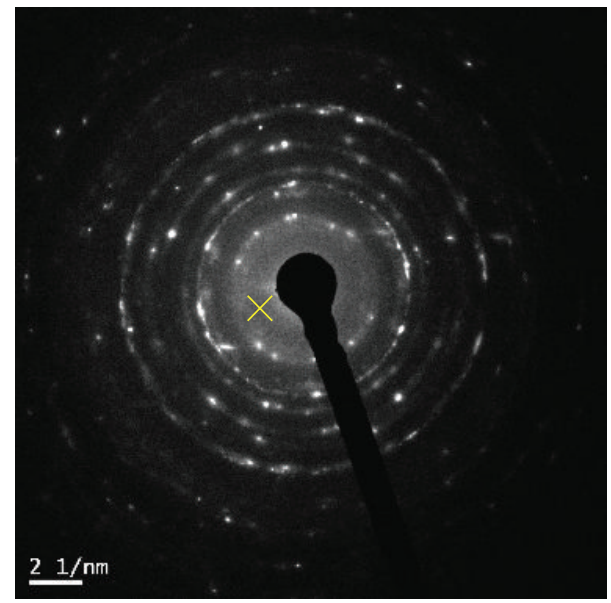

(f)

Figure 1: ((a), (b), and (c)) TEM images of the HNM particles. In (d), the "legs" (fibers) are shown on an expanded scale. (e) A HR-TEM image, taken with atomic resolution at the outer part of a particle. Oval curves (yellow) mark crystalline domains. (f) The electron diffraction pattern. The yellow cross denotes the amorphous ring that is superimposed on the crystalline reflections. 


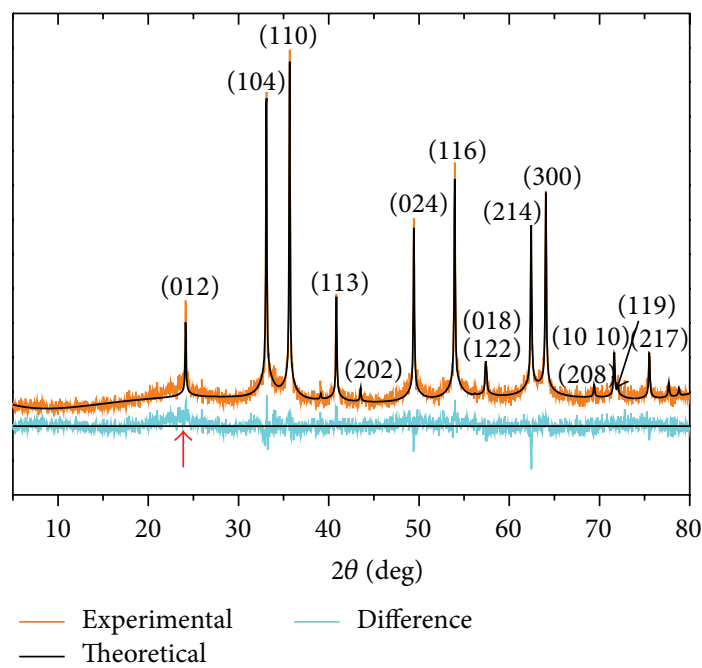

(a)

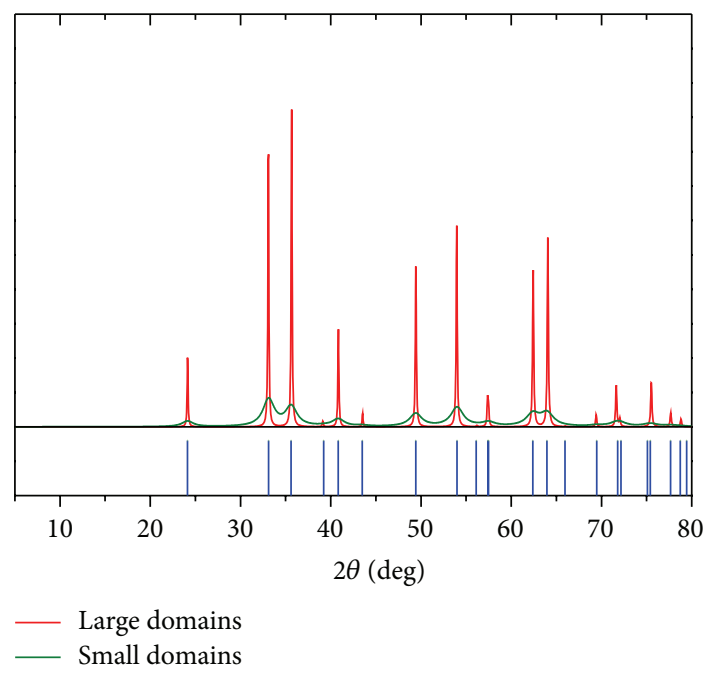

(b)

FIGURE 2: (a) The experimental XRD pattern (orange trace) of the synthesized product consisting of the HNM particles and the small particles observed as a background in Figure 1(c). The total theoretical pattern of the HNM particles is shown by a black curve, whereas the difference is shown by the cyan trace. The arrow indicates the broad amorphous peak. (b) The theoretical XRD patterns of the two phases that constitute the HNM particles (both hematites with the same size of the unit cell, but different size of the crystalline domains). The narrow peaks (red curve) are associated with large crystalline domains, whereas the broad peaks (green curve) describe small domains. The calculated peak positions from the fitted model are shown as a stick pattern at the bottom.

of $6 \pm 0.5 \mathrm{~nm}$. This is in good agreement with the qualitative estimate from the HR-TEM images (Figure 1(e)), where such a bimodal distribution of the crystalline domains was directly observed. The combination of XRD and HR-TEM analysis thus yields the conclusion that the HNM particles are pure hematite, composed of a spherical-like central body of about $100 \mathrm{~nm}$ diameter, which is crystalline in the interior (average crystalline domain size of $89 \mathrm{~nm}$ ), whereas the outer part of the body and the "legs" (fibers) contain small crystalline domains of $6 \mathrm{~nm}$ average size.

The question whether the central body of the nanoparticles is indeed a uniform spherical-like crystalline domain or it is a stack of thin crystalline plates (discs) was addressed by considering the dependence of the XRD peak broadening on the Miller indices. Hematite often grows in the morphology of thin disks, where disks of diameters less than $100 \mathrm{~nm}$ show better crystal development in the $a$ basal plane than along the $c$ (hexagonal) direction. This can be recognized from differential X-ray peak broadening with the $h k 0$ peaks being sharper than the $h k l$ peaks [1]. No differential broadening was observed in the XRD pattern of the HNM particles, thus excluding the possibility that the nanoparticles' body could be a stack of thin disks.

The role of silicon in the formation of the peculiar "nanomedusa" type morphology is an open question. All peaks observed in the XRD pattern belong to hematite and there are no additional peaks that would remain unindexed. This indicates that silicon did not enter the crystalline structure. Silicon is also not present in the elemental state or in the form of some crystalline compound. Amorphous silicon cannot be excluded. The presence of silicon in the HNM particles was checked by performing an EDS line scan and element mapping images. The EDS line scan is shown in Figure 3. The large spherical-like particle in the left part of the trace is an HNM particle, whereas the smaller particles in the right part of the trace are the nanoparticles observed in the TEM image (Figure 1(c)) as a background to the HNM particles. The distributions of the $\mathrm{Fe}, \mathrm{Si}$, and $\mathrm{O}$ elements along the trace are shown in separate panels, demonstrating that the small particles contain only $\mathrm{Si}$ and O. Since the XRD pattern shows only the peaks of pure hematite, small particles are very likely amorphous $\mathrm{SiO}_{2}$. According to literature [6], the XRD pattern of amorphous $\mathrm{SiO}_{2}$ shows a weak broad peak at $2 \theta$ between $20^{\circ}$ and $25^{\circ}$ due to short-range order of the local environment. Such broad peak is indeed observed in the XRD pattern of Figure 2(a), being more clearly visible in the difference (cyan) trace, where it is marked by an arrow. As already discussed, the electron diffraction pattern of Figure 1(f) also shows amorphous pattern superimposed on the sharp crystalline reflections, thus supporting the presence of amorphous $\mathrm{SiO}_{2}$. However, the amorphous interfaces between the crystalline regions in the HNM particles are expected to contribute to the amorphous pattern as well. The Fe and Si elemental maps over the area including many HNM particles are shown in Figure 4. The upper panel of Figure 4 shows a dark-field image of the HNM particles, whereas the two bottom panels show the distribution of $\mathrm{Fe}$ and $\mathrm{Si}$ elements over the scanned area. Silicon is detected on all particles, being very likely adsorbed on their surface in the form of $\mathrm{SiO}_{2}$. Though the role of silicon in the "nanomedusa" morphology formation is unclear, adsorbed $\mathrm{SiO}_{2}$ molecules may inhibit crystal growth, resulting in a nonuniform growth of the HNM particles in certain directions.

3.2. Magnetic Properties. Hematite is a magnetic material that shows interesting magnetism $[1,7]$. Bulk hematite is weakly ferromagnetic (FM) between the Néel temperature $T_{N} \approx$ 


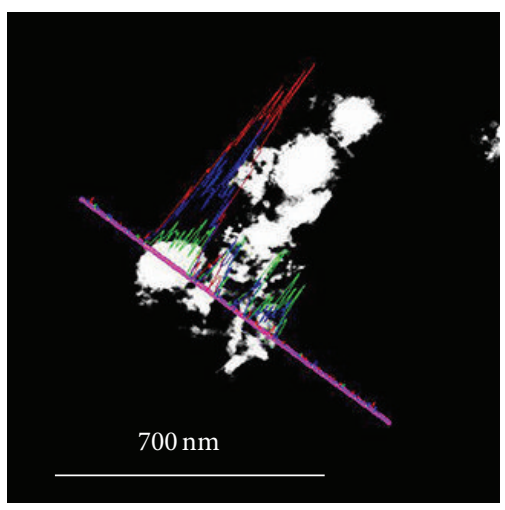

(a)
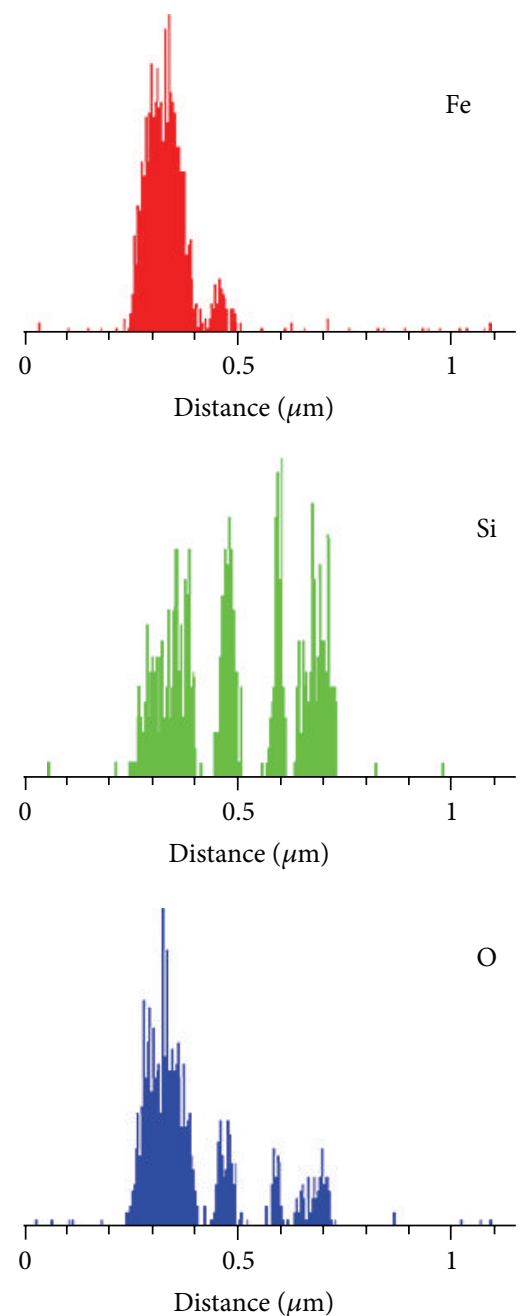

(b)

FIGURE 3: An EDS line scan (a) over the area containing an HNM particle in the left part of the trace and smaller particles in the right part of the trace, which are the "background" particles observed in the TEM image of Figure 1(c). (b) shows the distributions of the Fe, $\mathrm{Si}$, and $\mathrm{O}$ elements along the trace.
$955 \mathrm{~K}$ and the Morin transition temperature $T_{M} \approx 263 \mathrm{~K}$. At $T_{M}$, a spin flip transition takes place to an antiferromagnetic (AFM) state. The magnetically ordered states possess the following spin structures. Above $T_{M}$, the $\mathrm{Fe}^{3+}$ ions are antiferromagnetically coupled across the shared faces of the $\mathrm{FeO}_{6}$ octahedra along the $c$-axis. In the basal plane, there are two interpenetrating AFM sublattices. As the magnetic moments of these sublattices are not exactly antiparallel (i.e., spin canted) with a canting angle of $<0.1^{\circ}$, a weak FM interaction results. At $T_{M}$, competition between the weak magnetoanisotropy of the $\mathrm{Fe}^{3+}$ ion and the dipolar anisotropy causes the magnetic moments to reorient from the basal plane at $T>T_{M}$ to an angle of $7^{\circ}$ to the $c$-axis at $T<T_{M}[8,9]$. In this state, the moments are exactly antiparallel and hematite is antiferromagnetic. For hematite in the nanoparticle morphology, these characteristics are affected by the particle size and shape. Spherical particles with diameter smaller than 25-30 $\mathrm{nm}$ are superparamagnetic [10] and the Morin temperature is lowered significantly or even suppressed [11-16].

Magnetic properties of the HNM particles were investigated by determining the magnetic susceptibility $\chi=M / H$ and the $M(H)$ curves, which measure the response of the spin system to an external magnetic field. A comparison to bulk hematite was made by using a commercial Alfa Aesar powder product of $99.998 \%$ purity. Figure 5(a) shows magnetic susceptibility $\chi$ of the HNM particles. Both zerofield-cooled (zfc) and field-cooled (fc) susceptibilities were determined in the field of 100 Oe. The zfc susceptibility is obtained by cooling the spin system to the lowest temperature in the absence of a magnetic field, where the magnetic field is applied and the susceptibility is measured in a subsequent heating run. The fc susceptibility is measured in a cooling run in the presence of a magnetic field, starting at the highest temperature. A magnetic phase transition is observed at the temperature $T_{N} \approx 930 \mathrm{~K}$, below which the magnetization grows as a power law $M \propto\left(T_{N}-T\right)^{\beta}$, typical of spontaneous magnetization in FM materials. The fit with $\beta=0.63$ in the region of the phase transition is shown in the inset of Figure $5(\mathrm{a}) . T_{N}$ of the HNM particles matches reasonably the Néel temperature of bulk hematite. Below the phase transition, $\chi_{\mathrm{zfc}}$ and $\chi_{\mathrm{fc}}$ both increase rapidly but show no difference $\left(\chi_{\mathrm{zfc}} \approx \chi_{\mathrm{fc}}\right)$ down to about $650 \mathrm{~K}$. Below that temperature, the $\mathrm{zfc}-\mathrm{fc}$ susceptibility splitting starts to be observed, demonstrating ergodicity breaking of the spin system. Upon further cooling, $\chi_{\mathrm{zfc}}-\chi_{\mathrm{fc}}$ splitting increases and becomes very large in the low-temperature range. The zfc and fc susceptibilities of bulk hematite (red curve in Figure 5(a)) were determined in the temperature range from 2 to $400 \mathrm{~K}$. The Morin transition from the weakly FM to the AFM state is observed around $260 \mathrm{~K}$ (marked by an arrow) and there is no $\mathrm{zfc}-\mathrm{fc}$ susceptibility splitting, except for the heating-cooling hysteresis in the region of the Morin transition.

The $M(H)$ curves of the HNM particles were determined at temperatures between 800 and $2 \mathrm{~K}$. A selected set of the $M(H)$ curves is shown in Figure 5(b). Hysteresis is observed in the temperature range below about $650 \mathrm{~K}$, where the susceptibility shows $\mathrm{zfc}-\mathrm{fc}$ splitting (in the nonergodic 


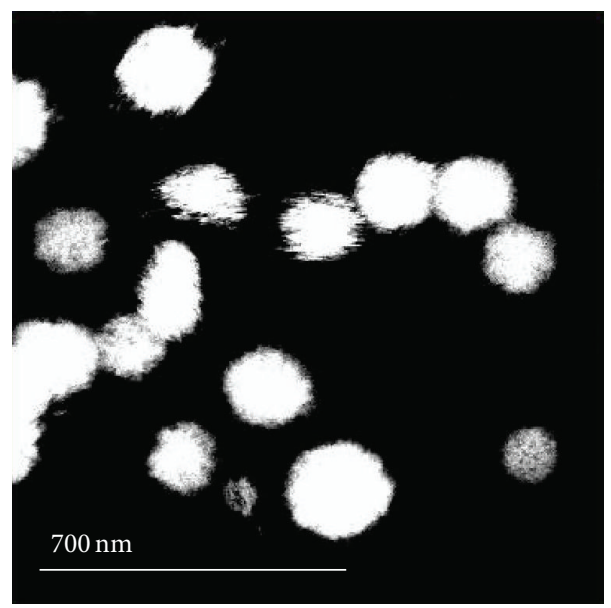

(a)

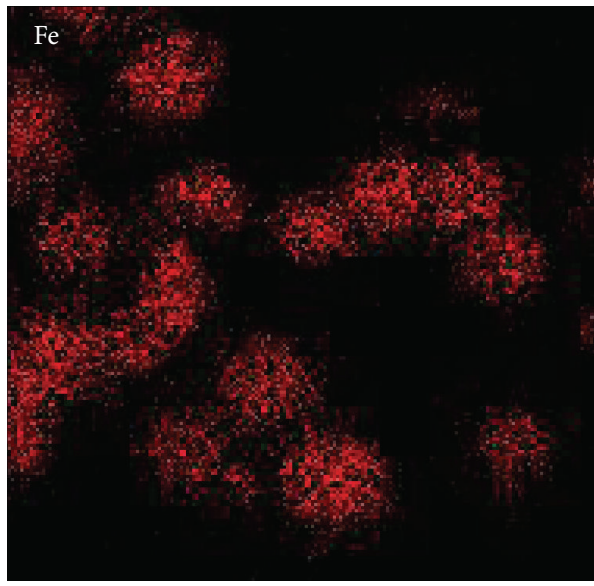

(b)

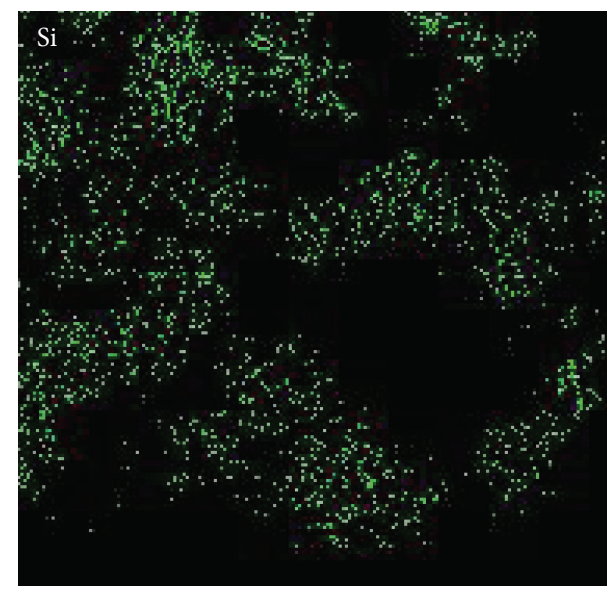

(c)

FIGURE 4: (a) shows a dark-field image of the HNM particles, whereas (b) and (c) show EDS distributions of the Fe and Si elements over the scanned area.

regime). The temperature dependence of the coercive field $H_{c}$ is shown in the inset of Figure $5(\mathrm{~b}) . H_{c}$ increases rapidly upon cooling and reaches the value of $600 \mathrm{Oe}$ at $T=2 \mathrm{~K}$. In the high-temperature range between the phase transition and $650 \mathrm{~K}$, the HNM particles show no hysteresis.

Figure 6(a) shows comparison of the $M(H)$ curve of the HNM particles at $T=2 \mathrm{~K}$ to the $M(H)$ curves of the bulk hematite at $T=300 \mathrm{~K}>T_{M}$ in the weakly FM state and at $T=$ $2 \mathrm{~K}<T_{M}$ in the AFM state. The weakly FM state of the bulk is characterized by a hysteresis loop in the low-field region due to the FM magnetization component, which has a coercive field of $H_{c}=2.5 \mathrm{kOe}$ and closes up in the field of about $8 \mathrm{kOe}$, whereas at higher fields the $M(H)$ relation becomes linear due to the AFM magnetization component. The $M(H)$ curve of the bulk hematite at $T<T_{M}$ is linear and shows no hysteresis, as typical for a bulk AFM state. The $M(H)$ curve of the HNM particles behaves differently, showing hysteretic behavior in the low-field region even at $T=2 \mathrm{~K}$. Away from the hysteretic region, the $M(H)$ relation shows curvature resembling the Brillouin function characteristic of localized paramagnetic moments with a tendency to approach a linear line in the high-field region. The low-field parts of the three $M(H)$ curves in the hysteretic region are shown on an expanded scale in the inset of Figure 6(a).

In order to unravel the magnetic state of the HNM particles, the shape of the $M(H)$ curve at $T=2 \mathrm{~K}$ was analyzed quantitatively. We assumed coexistence of three different components in the total magnetization at this low temperature, which can be written as

$$
M=M_{S} L\left(\frac{\mu_{\mathrm{FM}} H}{k_{B} T}\right)+\chi_{\mathrm{AFM}} H+M_{0} B_{J}\left(\frac{\mu H}{k_{B} T}\right) .
$$

The first term on the right describes the FM magnetization component. The average field-dependence of the FM term can be conveniently reproduced by the Langevin function $L(y)=\operatorname{coth}(y)-1 / y$, assuming unphysically large magnetic moments ("superspins") $\mu_{\mathrm{FM}}=x \mu_{B}$, where $\mu_{B}$ is the Bohr magneton and $x \gg 1$. The Langevin function cannot describe the FM hysteresis but can reproduce the fast average increase of the magnetization in the low-field region and its saturation to a plateau at high fields, determined by the FM saturation 


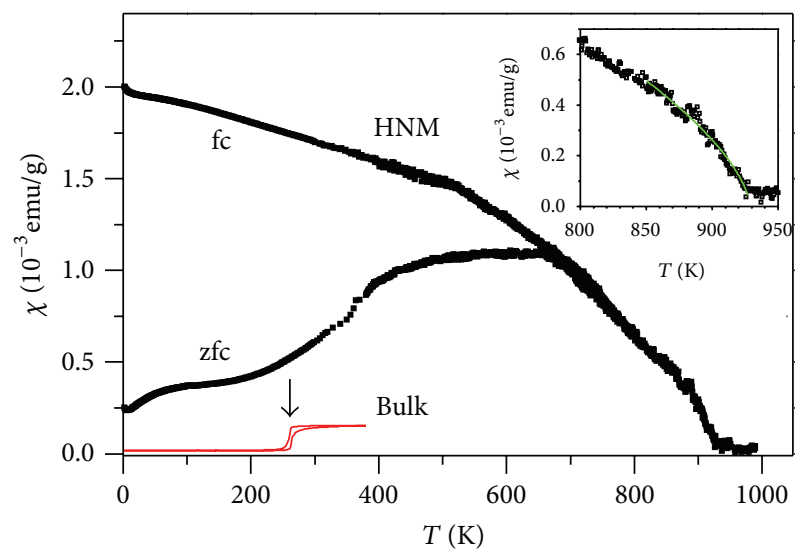

(a)

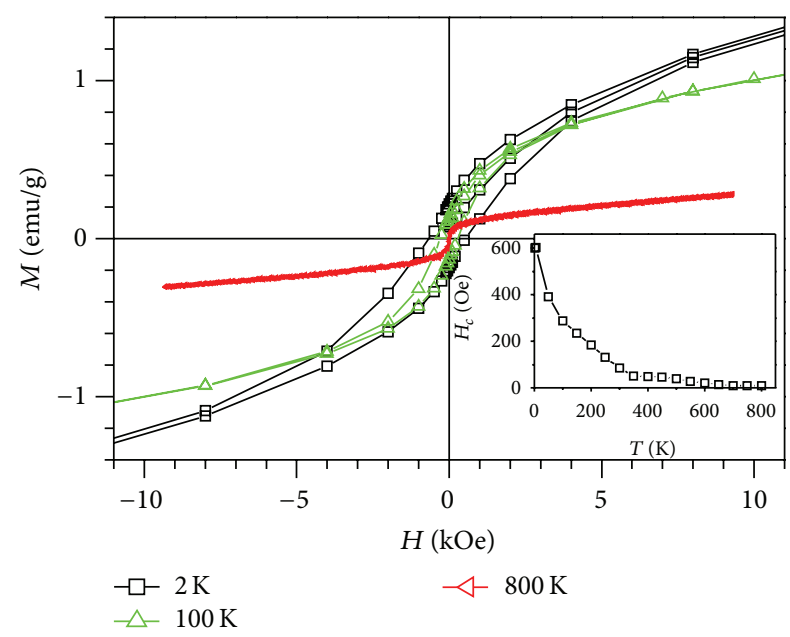

(b)

FIgURE 5: (a) Zfc and fc magnetic susceptibilities of the HNM particles in the temperature range between 1000 and $2 \mathrm{~K}$ in a magnetic field of 100 Oe. The inset shows the fit of the experimental data with the equation $M=A\left(T_{N}-T\right)^{\beta}$ (green curve) in the region of the phase transition. The $\mathrm{zfc}$ and $\mathrm{fc}$ susceptibilities of bulk hematite in the temperature range from 2 to $400 \mathrm{~K}$ are shown by the red curves. The Morin transition is marked by an arrow. (b) A selected set of $M(H)$ curves of the HNM particles between 800 and $2 \mathrm{~K}$. The inset shows temperature dependence of the coercive field $H_{c}$.

magnetization $M_{S}$. The second term, $\chi_{\mathrm{AFM}} H$, describes the AFM magnetization component, where $\chi_{\mathrm{AFM}}$ is the antiferromagnetic susceptibility. The third term is the paramagnetic magnetization component, where $M_{0}$ is the paramagnetic saturation magnetization and $B_{J}$ is the Brillouin function. This term describes the response of localized free magnetic moments $\mu=J g \mu_{B}$ to the external magnetic field, where $J$ is the angular momentum and $g$ is the Landé factor (taken as $g=2$ ). It is important to note that although the model of (1) separates the total magnetization into the FM, AFM, and paramagnetic components, it does not assume the existence of separated FM, AFM, and paramagnetic spin domains in the HNM particles. In the fit procedure, we have assumed an $\mathrm{Fe}^{3+}$ ionization state $(J=5 / 2)$ and obtained the fit parameters $\mu_{\mathrm{FM}}=66 \mu_{B}$ and $M_{S}=0.81 \mathrm{emu} / \mathrm{g}$ for the FM term, $\chi_{\mathrm{AFM}}=1.35 \times 10^{-5} \mathrm{emu} /(\mathrm{g} \cdot \mathrm{Oe})$ for the AFM term, and $M_{0}=1.41 \mathrm{emu} / \mathrm{g}$ for the paramagnetic term. The fit with (1) is shown in Figure 6(b) by a black curve, reproducing well the experimental data (except the hysteresis). The theoretical FM, AFM, and paramagnetic contributions are shown separately as well. In the inset of Figure 6(b) we plot the experimental FM component only, obtained by subtracting the theoretical AFM and paramagnetic terms from the total experimental magnetization, $M_{\mathrm{FM}}=M-\chi_{\mathrm{AFM}} H-M_{0} B_{J}$. The FM hysteresis loop is now more clearly observed, showing that it closes up in the field of about $8 \mathrm{kOe}$ (thus at the same field value as the FM loop of the bulk hematite in the weakly FM state at $300 \mathrm{~K}$ ), but the coercive field of $600 \mathrm{Oe}$ is much smaller than the coercive field of the weakly FM state in the bulk that amounts to $2.5 \mathrm{kOe}$ at $300 \mathrm{~K}$.

The above-presented experiments and analysis suggest the following magnetic state of the HNM particles at $T=2 \mathrm{~K}$. The $M(H)$ hysteresis loop that closes up in a field of about $8 \mathrm{kOe}$ is typical ferromagnetic (recall that an AFM hysteresis loop due to uncompensated spin fraction usually closes up in a much larger field of several $10 \mathrm{kOe}$ ). The coexistence of the FM and AFM magnetization components indicates weakly FM state, similar to the bulk hematite in the hightemperature range between the Néel temperature $T_{N}$ and the Morin transition temperature $T_{M}$. The Morin transition is absent in the HNM particles, so that the weakly FM state is retained down to $2 \mathrm{~K}$, at least. The weakly FM ordered regions can be associated with the large crystalline domains of $89 \mathrm{~nm}$ average size (the HNM particles' cores), as determined from the HR-TEM images and XRD analysis.

The paramagnetic component of the magnetization, which is observed in addition to the FM and AFM components, originates from two sources. A part of it can be associated with the amorphous interfaces between the crystalline domains, where the interactions between randomly distributed $\mathrm{Fe}^{3+}$ moments do not lead to magnetic ordering. The second part originates from the small crystalline domains of $6 \mathrm{~nm}$ average size, which should be superparamagnetic according to the literature reports. In the HNM particles, their magnetic signal combines with the paramagnetic signal from the amorphous interfaces and the two contributions cannot be unambiguously resolved experimentally.

The high-temperature region between $T_{N}$ and about $650 \mathrm{~K}$, where the $\mathrm{zfc}-\mathrm{fc}$ susceptibility splitting and the $M(H)$ hysteresis are absent, deserves special attention. This phenomenon can be explained by considering that the basal plane of the hematite structure has sixfold symmetry, so that the FM magnetization has six equivalent easy directions in this plane. Fast thermally assisted jumping of spins between the six easy directions in the basal plane polarizes spin domains into the direction of the external field and restores ergodicity at high temperatures, causing the $\chi_{\mathrm{zfc}}-$ $\chi_{\mathrm{fc}}$ difference and the $M(H)$ hysteresis to vanish there. This indicates that upon crossing $T_{N}$, the HNM particles' crystalline cores first enter a superparamagnetic regime, which then gradually transforms into the weakly FM state upon lowering the temperature, once the thermal energy for the spin reorientations is insufficient to induce frequent 


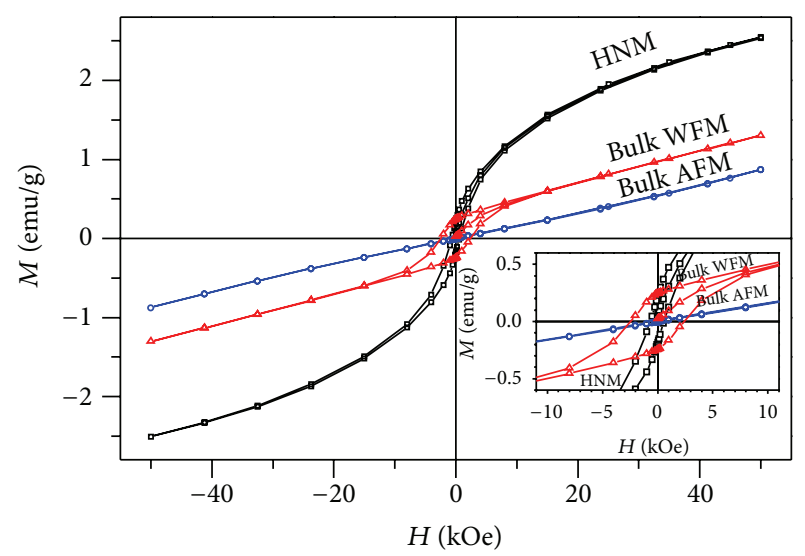

(a)

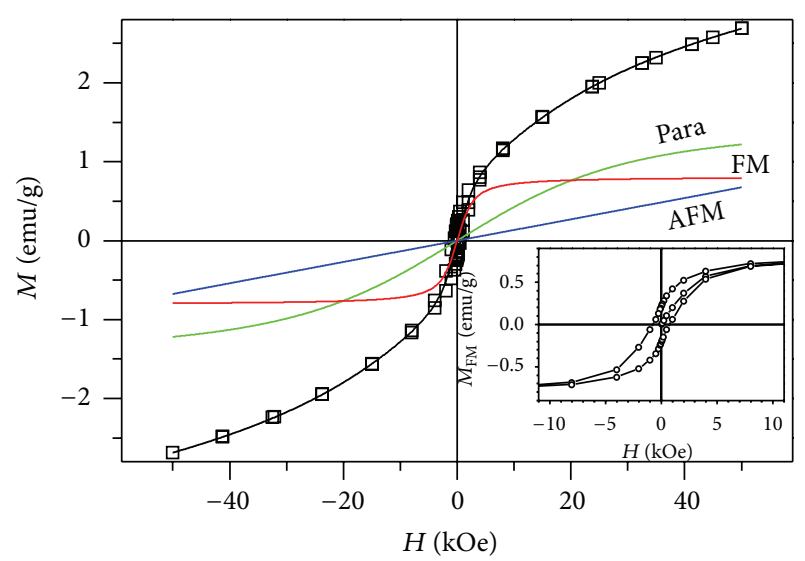

(b)

FIGURE 6: (a) A comparison of the $M(H)$ curve of the HNM particles at $T=2 \mathrm{~K}$ to the $M(H)$ curves of the bulk hematite at $T=300 \mathrm{~K}>$ $T_{M}$ in the weakly FM state (the "bulk WFM" curve) and at $T=$ $2 \mathrm{~K}<T_{M}$ in the AFM state (the "bulk AFM" curve). The $M(H)$ curves in the hysteretic region are shown on an expanded scale in the inset. (b) The $M(H)$ curve of the HNM particles at $T=2 \mathrm{~K}$. The fit with (1) is shown by a black curve. The theoretical FM, AFM, and paramagnetic contributions are shown separately as well. The inset shows the experimental FM component only, obtained by subtracting the theoretical AFM and paramagnetic terms from the total experimental magnetization, $M_{\mathrm{FM}}=M-\chi_{\mathrm{AFM}} H-M_{0} B_{J}$.

jumps of the magnetization between the six easy directions in the basal plane.

A quantitative difference between the weakly FM states of the HNM particles and the bulk hematite can be deduced from their $M(H)$ hysteresis loops. For both materials, the loops close in the same field of $8 \mathrm{kOe}$. However, the coercive field of the HNM particles $H_{c}=600 \mathrm{Oe}$ at $2 \mathrm{~K}$ is much smaller than the coercive field $H_{c}=2.5 \mathrm{kOe}$ at $300 \mathrm{~K}$ of the bulk, showing that the weakly FM spin structure in the HNM particles is magnetically considerably "softer" than in the bulk, owing to a reduced strength of the interspin interactions caused by nanodimensions of the crystalline regions and surface effects, where spin coordination by the neighbors is reduced.

\section{Conclusions}

In conclusion, we have synthesized hematite particles in a peculiar "nanomedusa" morphology using a hydrothermal synthesis method. The "hairy" particles consist of a sphericallike core of about $100 \mathrm{~nm}$ diameter and fibrous exterior composed of thin "legs" of $5 \mathrm{~nm}$ diameter grown along one preferential direction. The particles' cores are crystalline and undergo a phase transition to a weakly FM state at a temperature of about $930 \mathrm{~K}$ that matches reasonably the Néel temperature of bulk hematite. However, unlike bulk hematite that undergoes Morin transition to the AFM state around room temperature and small hematite nanoparticles that are superparamagnetic, the HNM particles remain weakly ferromagnetic down to the lowest investigated temperature of $2 \mathrm{~K}$. Each HNM particle thus represents a nanodimensional ferromagnet in a very broad temperature interval, extending much above the room temperature. Such high-temperature ferromagnetic nanoparticles are not frequently found among the nanomaterials. Due to the weakly FM character and biocompatibility, the HNM particles may find application in various branches of medicine and biology.

\section{Conflict of Interests}

The authors declare that there is no conflict of interests regarding the publication of this paper.

\section{References}

[1] R. M. Cornell and U. Schwertmann, The Iron Oxides. Structure, Properties, Occurrences and Uses, VCH, Weinheim, Germany, 1996.

[2] S. Hamada and E. Matijević, "Formation of monodispersed colloidal cubic haematite particles in ethanol + water solutions," Journal of the Chemical Society, Faraday Transactions 1: Physical Chemistry in Condensed Phases, vol. 78, no. 7, pp. 2147-2156, 1982.

[3] E. Matijević and Š. Cimaš, "Formation of uniform colloidal iron (III) oxides in ethylene glycol-water solutions," Colloid and Polymer Science, vol. 265, no. 2, pp. 155-163, 1987.

[4] V. A. Sadykov, L. A. Isupova, S. V. Tsybulya et al., "Effect of mechanical activation on the real structure and reactivity of iron (III) oxide with corundum-type structure," Journal of Solid State Chemistry, vol. 123, no. 2, pp. 191-202, 1996.

[5] R. W. Cheary and A. Coelho, "Fundamental parameters approach to X-ray line-profile fitting," Journal of Applied Crystallography, vol. 25, no. 2, pp. 109-121, 1992.

[6] N. Yan, F. Wang, H. Zhong et al., "Hollow Porous $\mathrm{SiO}_{2}$ nanocubes towards high-performance anodes for lithium-ion batteries," Scientific Reports, vol. 3, article 1568, 2013.

[7] A. H. Morrish, Canted Antiferromagnetism: Hematite, World Scientific Publishing, Singapore, 1994.

[8] A. H. Morrish, G. B. Johnston, and N. A. Curry, "Magnetic transition in pure and Ga doped $\alpha-\mathrm{Fe}_{2} \mathrm{O}_{3}$," Physics Letters, vol. 7, no. 3, pp. 177-178, 1963.

[9] J. O. Artman, J. C. Murphy, and S. Foner, "Magnetic anisotropy in antiferromagnetic corundum-type sesquioxides," Physical Review, vol. 138, no. 3, pp. A912-A917, 1965. 
[10] D. J. Dunlop and Ö. Özdemir, Rock Magnetism: Fundamentals and Frontiers, Cambridge University Press, Cambridge, UK, 1997.

[11] D. Schroeer and R. C. Nininger, "Morin transition in $\alpha-\mathrm{Fe}_{2} \mathrm{O}_{3}$ microcyrstals," Physical Review Letters, vol. 19, no. 11, pp. 632634, 1967.

[12] N. Yamamoto, "The shift of the spin flip temperature of $\alpha-\mathrm{Fe}_{2} \mathrm{O}_{3}$ fine particles," Journal of the Physical Society of Japan, vol. 24, no. 1, pp. 23-28, 1968.

[13] R. C. Nininger Jr. and D. Schroeer, "Mössbauer studies of the morin transition in bulk and microcrystalline $\alpha-\mathrm{Fe}_{2} \mathrm{O}_{3}$," Journal of Physics and Chemistry of Solids, vol. 39, no. 2, pp. 137-144, 1978.

[14] J. Muench, S. Arajs, and E. Matijevic, "The Morin transition in small $\alpha-\mathrm{Fe}_{2} \mathrm{O}_{3}$ particles," Physica Status Solidi A, vol. 92, pp. 187192, 1985.

[15] N. Amin and S. Arajs, "Morin temperature of annealed submicronic $\alpha-\mathrm{F}_{2} \mathrm{O}_{3}$ particles," Physical Review B, vol. 35, no. 10, pp. 4810-4811, 1987.

[16] F. Bødker, M. F. Hansen, C. B. Koch, K. Lefmann, and S. Mørup, "Magnetic properties of hematite nanoparticles," Physical Review B, vol. 61, no. 10, pp. 6826-6838, 2000. 

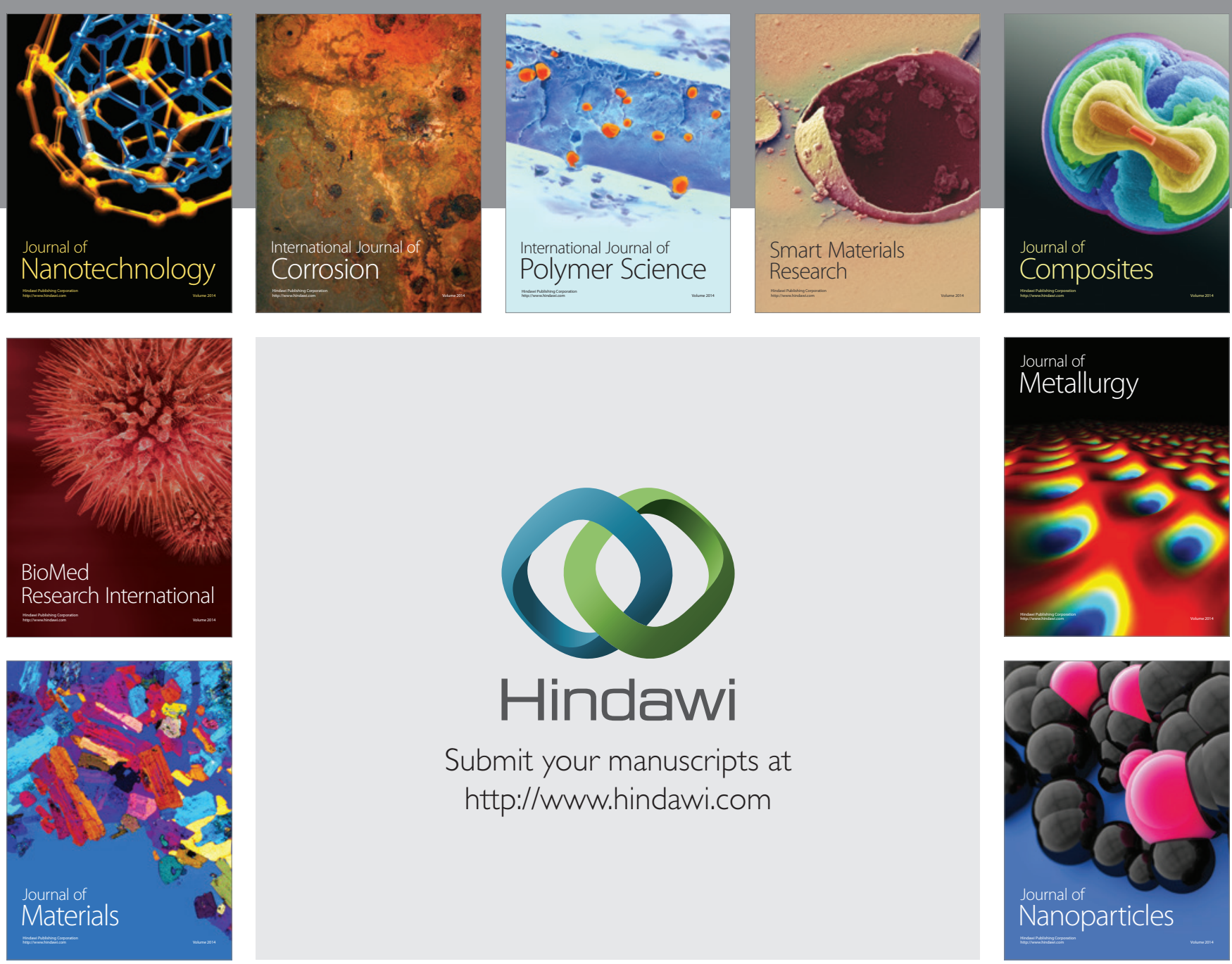

Submit your manuscripts at http://www.hindawi.com
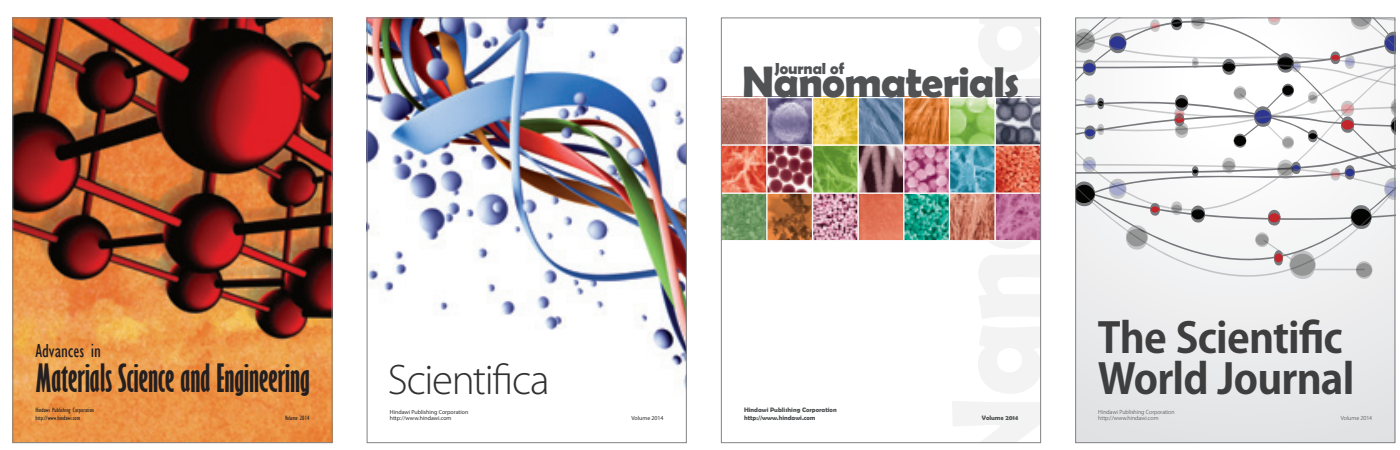

\section{The Scientific World Journal}
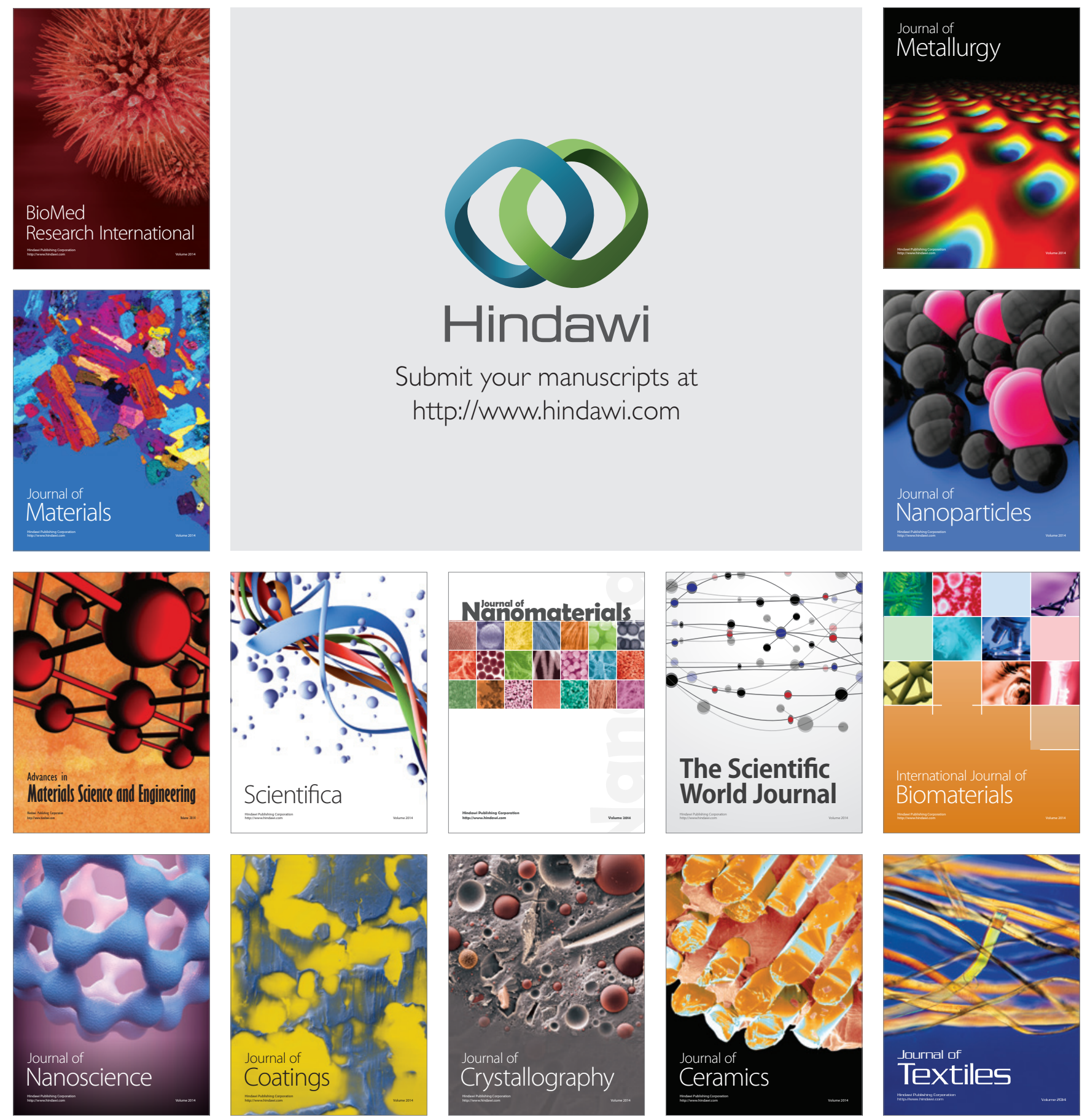